\title{
Association Of Serial Beta HCG And Progesterone Level With Outcome In Pregnancy Of Unknown Location
}

\author{
Ayesha Ajmi \\ ABSTRACT \\ Objective: To study the association of change in serial beta HCG level over 48 hours and serum progesterone with \\ final diagnosis i.e. viable intrauterine pregnancy, ectopic pregnancy or failing pregnancy in cases initially labelled as \\ pregnancy of unknown location.
}

Study Design: prospective population based study

Place and duration of study: Early Pregnancy Assessment unit of Homerton University Hospital London from December 2013 to February 2014

Methodology: Fifty patients were recruited in the study who presented to early pregnancy assessment unit and had positive urine for pregnancy test but no evidence of pregnancy on transvaginal scan. Initial beta HCG progesterone and transvaginal scan were done in all cases. Patients were followed up with repeat HCG at 48 hour interval and repeat TVS until final diagnosis was established.

Results: Final diagnosis was miscarriage $58 \%$, viable intrauterine pregnancy $24 \%$ and ectopic pregnancy $12 \% .67 \%$ of patients with rise in HCG $>60 \%$ had viable intrauterine pregnancy whereas all patients with $>50 \%$ fall in HCG had a miscarriage. A highly significant association of $>60 \%$ rise of HCG with viable intrauterine pregnancy and of $>50 \%$ fall in HCG with miscarriage was observed with $p$-value $<0.0001$.

$58 \%$ of patients with progesterone $>30$ had viable intrauterine pregnancy whereas $83 \%$ of patients with progesterone $<10$ were miscarriage and $17 \%$ had ectopic pregnancy. A highly significant association of final diagnosis of viable intrauterine pregnancy and progesterone level $>30$ was observed with p-value $<0.0001$.

Conclusion: Although there is high association of $>60 \%$ rise in 48 hour repeat HCG and progesterone $>30$ with viable intrauterine pregnancy, ectopic pregnancy cannot be ruled out on the basis of biochemical test. Therefore a high index of suspicion is required to diagnose cases of ectopic pregnancy using clinical signs and symptoms, transvaginal scan as well as biochemical tests such as serial beta HCG and progesterone levels.

Key words: Pregnancy of unknown location, Ectopic pregnancy, serial beta HCG Progesterone

\section{INTRODUCTION}

Bleeding and/ or pain in early pregnancy is one of the commonest presenting complaints in gynaecology. Pregnancy of unknown location is defined as pregnancy in which urine for pregnancy test is positive but pregnancy, either intrauterine or extrauterine is not visible on transvaginal ultrasound $\operatorname{scan}^{1} .20 \%$ of pregnancies may initially be classified as pregnancy of unknown location (PUL). PUL is not a diagnosis, rather it is a clinical query that needs to be solved. The final outcomes may be a viable intrauterine pregnancy, failing pregnancy of unknown location (miscarriage) or ectopic pregnancy ${ }^{1}$.

The commonest outcome of cases initially labelled as pregnancy of unknown location is failing pregnancy that may be intrauterine or extrauterine and resolves

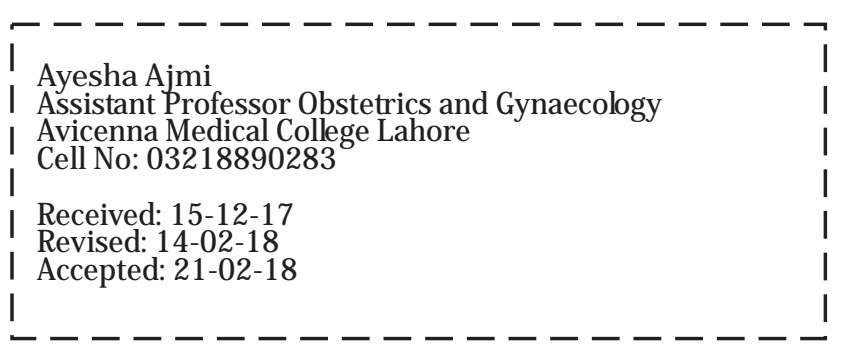

spontaneously. Another common outcome is viable intrauterine pregnancy. The most feared outcome is ectopic pregnancy. It may be tempting to diagnose a case of heavy bleeding with empty uterus as clear case of miscarriage but it can be misleading and there has been a case of maternal death with such presentation being managed as miscarriage which turned out to be ruptured ectopic in future ${ }^{3}$. Therefore careful follow up of pregnant women with empty uterus and positive pregnancy test is mandatory to avoid early intervention in case of viable intrauterine pregnancy and not to miss ectopic pregnancy. PUL is not synonymous with ectopic pregnancy but it should be considered as high risk of ectopic until and unless proved otherwise ${ }^{4}$.

Serum HCG is merely an indicator of pregnancy while change in HCG over 48 hours is useful in distinguishing failing PUL from intrauterine and ectopic pregnancy. This is particularly the case when HCG is less than the discriminatory zone (HCGlevel above which it is expected to see an intrauterine pregnancy, taken to be 1000- 2400 depending on ultrasound equipment and sonographer expertise $)^{5}$.

The minimum rise for a potentially viable pregnancy that 
presents with vaginal bleeding and/ or pain is $53 \%$ at 48 hour interval ${ }^{6}$. If HCG falls by at least $15 \%$ the most likely outcome is failing pregnancy. When the rise or fall in HCG is suboptimal, the most likely outcome is ectopic pregnancy. However some ectopics can mimic the rise as in viable intrauterine pregnancy and others can mimic the fall as in miscarriage ${ }^{7}$.

Serum progesterone is a good predictor of viability of pregnancy but a poor predictor of location of pregnancy. Level $<20 \mathrm{nmol} / \mathrm{l}$ is most likely associated with failing pregnancy whilst levels $>25$ are likely to predict and $>60$ are strongly associated with viable pregnancy. A one off serum progesterone level may be a useful adjunct in the management of $\mathrm{PUL}^{8}$.

PUL poses a diagnostic challenge. Every case should be managed according to clinical signs and symptoms. Transvaginal ultrasound is gold standard and cornerstone in diagnosis and serial HCG and serum progesterone are useful adjuncts in diagnosis and to guide management. Most cases can be managed conservatively. A high index of suspicion is required so that ectopic pregnancy is not missed. At the same time unnecessary intervention should be avoided should it be early case of viable intrauterine pregnancy or self resolving failing pregnancy ${ }^{9}$. Senior gynaecologist's involvement in decision making is of paramount importance.

\section{METHODOLOGY:}

The study was carried out in Early Pregnancy Assessment Unit (EPAU) of Homerton University Hospital London UK over 3 months period from December 2013 to February 2014. 50 patients were recruited who presented with abdominal pain or PV bleeding or both. Repeat HCG was done in $92 \%$ cases, $4 \%$ were diagnosed with miscarriage on the basis of history and very low initial HCG level and another $4 \%$ were lost to follow up. Repeat TVS was done in $46 \%$ of cases. Final diagnosis was established in light of signs and symptoms, scan findings, percentage rise or fall in HCG at 48hour interval and serum progesterone level. Final diagnosis was miscarriage, viable pregnancy or ectopic pregnancy. Data was analysed on SPSS and association of percentage change in serial beta HCG over 48 hours and serum progesterone level with final diagnosis was evaluated.

\section{RESULTS:}

In this study, the age of 34 (68.0\%) patients was in 2035 years age category, whereas $14(28.0 \%)$ patients were $>35$ years old and only $2(4.0 \%)$ patients were $<20$ years old. Among all 50 patients, 9 (18.0\%) were nulliparous, $28(56.0 \%)$ were multiparous and parity was not known for $13(26 \%)$. The past history of ectopic pregnancy was present in $6(12.0 \%)$ patients, absent in $35(70.0 \%)$ patients and not known in $9(18.0 \%)$ patients. The amenorrhea was reported to be for $<5$ weeks in $5(10.0 \%)$ patients, $5-6$ weeks in $22(44.0 \%)$ patients, $>6$ weeks in $17(34.0 \%)$ patients and was not surely known for 6 (12.0\%) patients. Among all 50 patients, 15 (30.0\%) presented with PV bleeding, 14 (28.0\%) with abdominal pain, 17 (34.0\%) with both PV bleeding and abdominal pain, $3(6.0 \%)$ came for reassurance scan and $1(2.0 \%)$ patients presented with pre TOP. The initial abdominal scan was done in $46(92.0 \%)$ patients, pseudosac was seen in $14(28.0 \%)$ patients and significant free fluid was observed in only $1(2.0 \%)$ patient.

The level of progesterone was observed to be $<10$ in 24 (48.0\%) patients, $10-30$ in 7(14.0\%) patients, and $>30$ in $19(38.0 \%)$ patients. The initial beta HCG level was $<1000$ in $36(72.0 \%)$ patients, $1000-2000$ in $10(20.0 \%)$ patients and $>2000$ in $4(8.0 \%)$ patients. Percentage rise or fall in 48 hour interval HCG is shown in table 2,

The diagnosis on repeat scan was PUL in $4(8.0 \%)$ patients, viable in $7(14.0 \%)$ patients, ectopic and miscarriage in $3(6.0 \%)$ patients each and uncertain viability in $6(12.0 \%)$. The final diagnosis was PUL in 3 $(6.0 \%)$ patients, viable in $12(24.0 \%)$ patients, ectopic in $3(6.0 \%)$ patients, miscarriage in $29(58.0 \%)$ patients and uncertain viability in none of the patients whereas $3(6.0 \%)$ patients were lost to follow up.

Final diagnosis was miscarriage $58 \%$, viable intrauterine pregnancy $24 \%$ and ectopic pregnancy $12 \%$. 33\% had more than $60 \%$ rise on repeat HCG of which $67 \%$ were finally diagnosed with viable pregnancy, $13 \%$ with miscarriage, $7 \%$ with ectopic pregnancy and $13 \%$ were lost to follow up. All (26\%) patients with more than 50\% fall in repeat HCG were diagnosed with miscarriage. $22 \%$ of total patients had $<50 \%$ fall in HCG of those $80 \%$ had miscarriage while $20 \%$ had ectopic. $20 \%$ had $\varangle 60 \%$ rise of which $11 \%$ were viable intrauterine pregnancy, $44 \%$ had miscarriage, $33 \%$ ectopic and $11 \%$ were lost to follow up. A highly significant association of $>60 \%$ rise of HCG with viable intrauterine pregnancy and of $>50 \%$ fall in HCG with miscarriage was observed with p-value $\varangle .0001$.

$58 \%$ of patients with progesterone $>30$ had viable intrauterine pregnancy. $16 \%$ ectopic and 11\% miscarriage, $15 \%$ lost to follow up. $83 \%$ of patients with progesterone $<10$ were miscarriage and $17 \%$ had ectopic. $88 \%$ of patients with progesterone between 10 and 30 had miscarriage and $12 \%$ had viable pregnancy.

A highly significant association of final diagnosis of viable intrauterine pregnancy and progesterone level $>30$ was observed with $\mathrm{p}$-value $<0.0001$.

\section{DISCUSSION:}

Diagnosis of a woman who initially presents with PUL may involve multiple visits to the hospital, blood tests, ultrasound and possible surgical procedures before definitive diagnosis can be made ${ }^{10}$. Initial Ultrasound is inconclusive in $40 \%$ of cases because pregnancy may be 


\begin{tabular}{|c|c|c|c|}
\hline & & Frequency & Percent \\
\hline \multirow{3}{*}{ Progesterone } & $<10$ & 24 & 48.0 \\
\hline & $10-30$ & 7 & 14.0 \\
\hline & $>30$ & 19 & 38.0 \\
\hline \multirow{3}{*}{ Initial Bhcg } & $<1000$ & 36 & 72.0 \\
\hline & $1000-2000$ & 10 & 20.0 \\
\hline & $>2000$ & 4 & 8.0 \\
\hline \multirow{5}{*}{$\begin{array}{l}\text { Percentage rise or fall } \\
\text { Repeat bhcg at } 48 \text { hours }\end{array}$} & $<60 \%$ rise & 9 & 20.0 \\
\hline & $<50 \%$ fall & 10 & 22.0 \\
\hline & $>50 \%$ fall & 12 & 26.0 \\
\hline & $>60 \%$ rise & 15 & 33.0 \\
\hline & NA & 4 & 8.0 \\
\hline
\end{tabular}

Table-1: The Level of Beta HCG and Progesterone Among Patients

\begin{tabular}{|l|c|c|c|c|}
\hline \multirow{2}{*}{} & \multicolumn{2}{|c|}{ Diagnosis Repeat Scan } & \multicolumn{2}{c|}{ Final Diagnosis } \\
\cline { 2 - 5 } & Frequency & Percent & Frequency & Percent \\
\hline PUL & 4 & 8.0 & 3 & 6.0 \\
\hline Viable & 7 & 14.0 & 12 & 24.0 \\
\hline Ectopic & 3 & 6.0 & 3 & 6.0 \\
\hline Miscarriage & 3 & 6.0 & 29 & 58.0 \\
\hline Uncertain viability & 6 & 12.0 & 0 & 0 \\
\hline NA & 27 & 54.0 & 0 & 0 \\
\hline Loss to follow up & 0 & 0 & 3 & 6.0 \\
\hline Total & 50 & 100.0 & & \\
\hline
\end{tabular}

Table-2: Repeat Scan and Final Diagnosis

\begin{tabular}{|l|c|c|c|c|c|c|}
\hline \multicolumn{2}{|c|}{ Final diagnosis } & Viable & Ectopic & Miscarriage & $\begin{array}{c}\text { Lost to } \\
\text { followup }\end{array}$ & P value \\
\hline \multirow{4}{*}{$\begin{array}{l}\text { Percentage rise or fall on } \\
\text { first repeat Beta HCG }\end{array}$} & $>60 \%$ rise & $67 \%$ & $7 \%$ & $13 \%$ & $13 \%$ & $11 \%$ \\
\cline { 2 - 7 } & $<60 \%$ rise & $11 \%$ & $33 \%$ & $44 \%$ & $0 \%$ & $<0.0001$ \\
\cline { 2 - 7 } & $>50 \%$ fall & $0 \%$ & $0 \%$ & $100 \%$ & $0 \%$ & $0 \%$ \\
\cline { 2 - 6 } & $<50 \%$ fall & $0 \%$ & $20 \%$ & $80 \%$ & $0 \%$ & $<0.0001$ \\
\cline { 2 - 6 } & $<10$ & $0 \%$ & $17 \%$ & $83 \%$ & $15 \%$ & \\
\hline
\end{tabular}

Table-3: Association of Percentage Rise or Fall of Beta HCG and Progestrone Levels with Final Diagnosis 
too small to be seen or gestational sac may have collapsed before being visualized. In the event of making diagnosis, an ectopic can rupture leading to intraabdominal haemorrhage. It is imperative to differentiate between ectopic, viable intrauterine pregnancy and failing pregnancy because treatment strategies differ and could impact on future fertility ${ }^{11}$. A serum biomarker can help in this differentiation, and the most commonly used biomarkers used in clinical practice are serial HCG and serum progesterone.

Single measurement of serum HCG is poor predictor of outcome of PUL. A recent meta analysis by Van Mello et al has confirmed this ${ }^{12}$. In contrast serial measurement of serum HCG offers good test for predicting viability. Kadar and Romero suggested in 1981 that minimal rate of increase in HCG over hours was $66 \%$ in a viable intrauterine pregnancy ${ }^{13}$.A subsequent study by Barnhart et al showed the minimum rise in HCG at 24 hours to be $24 \%$ and at 48 ours to be $53 \% .{ }^{14}$ Seeber proposed minimal rise of $35 \%$ consistent with viable pregnancy. ${ }^{15}$ Most units in UK use rise in HCG 50-66\% to indicate increase in HCG compatible with viable pregnancy. Some ectopic pregnancies also demonstrate a normal rise in HCG

A decrease in HCG of $>13 \%$ has a sensitivity of $92.7 \%$ and specificity of $96.7 \%$ for predicting a failing PUL ${ }^{16}$. Unfortunately there is no single method to characterize the pattern of HCG change over48 hours in cases of PUL subsequently diagnosed as ectopic pregnancy. Majority would have serum HCG rise slower than would be expected in intrauterine viable pregnancy or decline slower than expected in a failing pregnancy. However $15-20 \%$ show rise similar to IUP and 10\% show pattern like failing $\mathrm{PUL}^{17}$.

Serum progesterone measurement at initial visit helps to reduce number of follow up visits. It is a marker of corpus luteum function and therefore will be high in viable pregnancies whether intrauterine or ectopic ${ }^{18}$. Progesterone $>25 \mathrm{nmol} / \mathrm{l}$ is likely to predict and $>60$ is highly associated with viable pregnancy ${ }^{19,20}$. Serum progesterone $<20$ is likely failing pregnancy and follow ups can be reduced ${ }^{21,22}$. Whether the failing PUL is ectopic or intrauterine is not of clinical importance. Hence progesterone is useful in identifying viability of pregnancy but not its location ${ }^{23,24}$. However its role is crucial in guiding the management of PUL and differentiating cases needing high or low level of suspicion ${ }^{25}$.

\section{CONCLUSION:}

Pregnancy of unknown location poses a diagnostic challenge. Most cases end up as failing pregnancy but it is important not to miss ectopic pregnancy that might rupture and at the same time not to rush to treat a self resolving pregnancy or terminate a normal pregnancy.
Serial HCG and progesterone are useful biomarkers that can be used as adjuncts to clinical judgement to guide the management of PUL.

\section{REFERENCES:}

1. Sagili H, Mohamed K. Pregnancy of unknown location: an evidence-based approach to management. The Obstetrician \& Gynaecologist 2008;10:224-230.

2. Schuneman, Wald TV; and Keith Hansen, MD, Pregnancy of unknown location, South Dakota Medicine, 2015;68(4): 163-167

3. O'HerlihyDeaths in early pregnancy, BJOG, 2011, vol. 118 Suppl. 1(1-203) In: Lewis G (ed.). Centre for Maternal and Child Enquiries (CMACE). Saving Mothers' Lives: Reviewing Maternal Deaths to Make Motherhood Safe 2006-2008. The Eighth Report on Confidential Enquiries into Maternal Deaths in the United Kin

4. Condous G, Okaro E, Khalid A et al. The accuracy of transvaginal ultrasonography for the diagnosis of ectopic pregnancy prior to surgery. Hum Reprod. 2005;20: 1404-1409

5. Condous G Timmerman D, Goldstein S, Valentin L, Jurkovic D, Bourne T. Pregnancies of unknown location: A consensus statement. Ultrasound Obstet Gynecol. 2006;28: 121-122.

6. Van Mello et al, Diagnostic value of serum HCG on the outcome of pregnancy of unknown location: a systematic review and meta-analysis, Hum Reprod Update, 2012; 18: 603-617

7. Barnhart, Sammel MD, Rinaudo PF, Ahou L, Hummel AC, Guo W. Symptomatic patients with an early viable intrauterine pregnancy: HCG curves redefined, Obstet Gynecol , 2004;104: 50-55

8. Condous G Kirk E, Van Calster B, Van Huffel S, Timmerman D, Bourne T. Failing pregnancies of unknown location: a prospective evaluation of the human chorionic gonadotrophin ratio, BJOG , 2006; 113: 521-527

9. Banerjee S, Aslam N, Woelfer B, Lawrence A, Elson J, Jurkovic D. Expectant management of early pregnancies of unknown location: a prospective evaluation of methods to predict spontaneous resolution of pregnancy, BJOG , 2001;108: 158-163

10. Cordina M, Schramm-Gajraj K, Ross JA, Lautman K, Jurkovic D. Introduction of a single visit protocol in the management of selected patients with pregnancy of unknown location: a prospective study, BJOG , 2011; 118: 693-697

11. Van Calster B, Abdallah Y, Guha S, Kirk E, Van Hoorde K, Condous G, Priesler J, Hoo W, Stalder C, Bottomley C, et al. Rationalizing the management of pregnancies of unknown location: temporal and external validation of a risk prediction model on 1962 pregnancies, Hum Reprod, 2013; 28: 609-616

12. E. Kirk, C. Bottomley, T. Bourne; Diagnosing ectopic pregnancy and current concepts in the management of pregnancy of unknown location, Human Reproduction Update, 2014;20(2): 250-26

13. Kadar N, Romero R. HCG assays and ectopic pregnancy, Lancet, 1981; 1:1205-06

14. Barnhart KT. Early pregnancy failure: beware of the pitfalls of modern management. Fertility and Sterilty. 2012;98: 1061-5. 
15. Seeber BE, Sammel MD, Guo W, Zhou L, Hummel A, Barnhart KT. Application of redefined human chorionic gonadotropin curves for the diagnosis of women at risk for ectopic pregnancy, Fertil Steril , 2006; 86: 454-459

16. Silva C, Sammel MD, Zhou L, Gracia C, Hummel AC, Barnhart K. Human chorionic gonadotrophin profile for women with ectopic pregnancy, Obstet Gynecol , 2006; 107: 605-610

17. Horne AW, McBride R, Denison FC. Normally rising HCG does not predict live birth in women presenting with pain and bleeding in early pregnancy, Eur J Obstet Gynecol Reprod Biol , 2011; 156: 120-121

18. Verhaegen J, Gallos ID, van Mello NM, Abdel-Aziz M, Takwoingi Y, Harb H, Deeks JJ, Mol BW, Coomarasamy A. Accuracy of single progesterone test to predict early pregnancy outcome in women with pain or bleeding: metaanalysis of cohort studies, BMJ , 2012; 345: 60-77

19. Royal College of Obstetricians and Gynaecologists, Green Top Guideline No. 25. The Management of Early Pregnancy Loss , 2006 London, UKRCOG Press
20. Goldstein S. Bourne T, Condous G. The diagnosis of miscarriage, Handbook of Early Pregnancy Care , 2006 UK Informa Health Care Ltd pg. 27-35

21. Brady, Paula C. MD. New Evidence to Guide Ectopic Pregnancy Diagnosis and Management, Obstetrical \& Gynecological Survey October 2017; 72(10):618-625.

22. Stulberg DB, Cain L, Dahlquist IH, et al. Ectopic pregnancy morbidity and mortality in low-income women, 2004-2008. Hum Reprod. 2016;31:666-671

23. Doubilet PM. Ultrasound evaluation of the first trimester. Radiol Clin North Am. 2014;52:1191-9.

24. Mavrelos D, Nicks H, Jamil A, et al. Efficacy and safety of a clinical protocol for expectant management of selected women diagnosed with a tubal ectopic pregnancy. Ultrasound Obstet Gynecol. 2013;42:102-107

25. Alkatout I, Honemeyer U, Strauss A, et al. Clinical diagnosis and treatment of ectopic pregnancy. Obstet Gynecol Surv. 2013;68:571-581 